\title{
Partial first-order logic relying on optimistic, pessimistic and average partial membership functions
}

\author{
Tamás Mihálydeák \\ Department of Computer Science, Faculty of Informatics, \\ University of Debrecen, Debrecen, Hungary \\ Email: mihalydeak.tamas@inf.unideb.hu
}

\begin{abstract}
One of the common features of decision-theoretic rough set models is that they rely on total background (available) knowledge in the sense that the knowledge covers the discourse universe. In the proposed framework the author gives up this requirement and allows that available knowledge about the discourse universe may be partial. It is shown by introducing optimistic, average and pessimistic partial membership functions that a decision-theoretic rough set model can be based on a very general version of partial approximation spaces. Different membership functions may serve as a base of the semantics of a partial first-order logic. The proposed logical system gives an exact possibility to introduce different semantic notions of logical consequence relations which can be used in order to make clear the consequences of our decisions.
\end{abstract}

Keywords: Approximation of sets, rough sets, decision-theoretic rough sets, partial first-order logic

\section{Introduction}

In last thirty years, a number of theoretical attempts have appeared in order to approximate sets. For example, rough set theory was originally proposed by Pawlak (see in [1], [2]), its different generalizations (see, e.g. in [3]) and granular computing ${ }^{1}$ play a crucial role in computer sciences. Rough set theory provides a powerful foundation to reveal and discover important structures and patterns in data, and to classify complex objects. ${ }^{2}$

In most cases, we have a family of base sets as subsets of a universe of discourse. In philosophy these sets represent our available knowledge, we consider them as the extensions of our available concepts/properties, and their members are the instances of these concepts/properties. The primary goal of different systems of set approximation is to "approximate/learn/express an unknown concept/property (represented by an arbitrary subset

\footnotetext{
${ }^{1}$ Rough set theory has served as a "pattern" of granular computing developments, see, e.g. in [4], [6], [7], [8], [9].

${ }^{2}$ An overview of some research trends on rough set foundations and rough set-based methods can be found in [13].
}

of the universe)" ([9] p. 520). A natural question may appear: What do we know about our available knowledge? If our available knowledge is represented by a family of properties of objects, then we can ask the following questions:

1. Does each object have at least one property from the given family?

2. Does each object have at best one property from the given family (or some of them have more than one)?

Pawlak's answers are 'yes' for both questions (so in his system each object has exactly one property from the given family), whereas covering rough set systems say 'yes' for the first question and no for the second one. A generalization of the theory of rough sets (see in [15], [16]) does not commit itself to answer 'yes' for any mentioned question: It does not suppose either the representations of properties belonging to mentioned family cover the discourse universe or the representations form a pairwise disjoint family of sets. From the approximation point of view the generalization can be considered as a system of partial approximation of sets.

Decision-theoretic rough set models can be considered as the probabilistic extensions of algebraic rough set models. Many papers deal with DTRS based on different systems of theory of rough sets (more details can be found, for example, in [5]). The main objectives of this paper are the followings:

1. to show that a decision-theoretic rough set model can be based on a very general version of partial approximation spaces by introducing optimistic, average and pessimistic partial membership functions relying on partial approximations of sets;

2. to present a partial first-order logic with precise semantics relying on different partial membership functions;

3 . to introduce different notions of logical consequence relations which can be used in order to make clear the consequences of our decisions.

After introducing the general partial approximation space as a generalization of different systems appeared in the theory of rough sets, three different 
types of partial membership functions are defined. They can be embedded in the semantics of partial first-order logic. At the end different notions of logical consequence are produced.

\section{General systems of tool-based approximation of sets}

In the following definition a most fundamental (and very general) notion of an approximation space is given. This core notion serves as the set-theoretical background of semantics of partial first-order logic relying on different partial membership functions.

Definition 1 The ordered 5-tuple $\left\langle U, \mathfrak{B}, \mathfrak{D}_{\mathfrak{B}}, \mathrm{I}, \mathrm{u}\right\rangle$ is a general partial approximation space with a Pawlakian approximation pair if

1. $U$ is a nonempty set;

2. $\mathfrak{B} \subseteq 2^{U} \backslash \emptyset, \mathfrak{B} \neq \emptyset$;

3. $\mathfrak{D}_{\mathfrak{B}}$ is an extension of $\mathfrak{B}$, and it is given by the following inductive definition:

(a) $\emptyset \in \mathfrak{D}_{\mathfrak{B}}$;

(b) if $D_{1}, D_{2} \in \mathfrak{D}_{\mathfrak{B}}$, then $D_{1} \cup D_{2} \in \mathfrak{D}_{\mathfrak{B}}$.

4. the functions $\mathbf{I}, \mathrm{u}$ form a Pawlakian approximation pair $\langle\mathrm{I}, \mathrm{u}\rangle$, i.e.

(a) $\mathrm{I}(S)=\bigcup \mathcal{C}^{\mathrm{I}}(S)$, where $\mathcal{C}^{\prime}(S)=\{B \mid B \in \mathfrak{B}$ and $B \subseteq S\}$;

(b) $\mathrm{u}(S)=\bigcup \mathcal{C}^{\mathrm{u}}(S)$, where $\mathcal{C}^{\mathrm{u}}(S)=\{B \mid B \in \mathfrak{B}$ and $B \cap S \neq \emptyset\}$.

Definition 2 If $\left\langle U, \mathfrak{B}, \mathfrak{D}_{\mathfrak{B}}, \mathrm{I}, \mathrm{u}\right\rangle$ is a general partial approximation space with a Pawlakian approximation pair and $S \subseteq U$, then $\mathrm{b}(S)=\bigcup\left(\mathcal{C}^{\mathrm{u}}(S) \backslash \mathcal{C}^{\mathrm{l}}(S)\right)$ is the border set of $S$.

Corollary 1 The followings hold for the approximation functions $\mathrm{I}, \mathrm{u}$ :

1. I, u : $2^{U} \rightarrow 2^{U}$;

2. $\mathrm{I}\left(2^{U}\right), \mathrm{u}\left(2^{U}\right) \subseteq \mathfrak{D}_{\mathfrak{B}}$ (definability of $\left.\mathrm{I}, \mathrm{u}\right)$;

3. the functions $\mathrm{I}$ and $\mathrm{u}$ are monotone, i.e. for all $S_{1}, S_{2} \in 2^{U}$ if $S_{1} \subseteq S_{2}$ then $\mathrm{I}\left(S_{1}\right) \subseteq \mathrm{I}\left(S_{2}\right)$ and $\mathrm{u}\left(S_{1}\right) \subseteq \mathrm{u}\left(S_{2}\right)$;

4. $\mathrm{u}(\emptyset)=\emptyset$ (normality of $\mathrm{u})$

5. if $S \in \mathfrak{D}_{\mathfrak{B}}$, then $\mathrm{I}(S)=S$ (granularity of $\mathfrak{D}_{\mathfrak{B}}$, i.e. I is standard);

6. if $S \in 2^{U}$, then $\mathrm{I}(S) \subseteq \mathrm{u}(S)$ (weak approximation property).

Definition 3 Let $\mathrm{GAS}=\left\langle U, \mathfrak{B}, \mathfrak{D}_{\mathfrak{B}}, \mathrm{l}, \mathrm{u}\right\rangle$ be a general approximation space and $S \subseteq U$.

1. If $S \in \mathfrak{D}_{\mathfrak{B}}$, then the set $S$ is definable in GAS.

2. If $S \in \mathfrak{D}_{\mathfrak{B}}$, and $U \backslash S \in \mathfrak{D}_{\mathfrak{B}}$ then the sets $S$ and $U \backslash S$ are totally definable in GAS.

Remark 2 Members of $\mathfrak{D}_{\mathfrak{B}}$ are definable, but not necessarily totally definable.

In a given general approximation space, the notions of well-approximated and totally wellapproximated sets can be introduced, and we call these sets crisp and totally crisp sets:
Definition 4 Let $\mathrm{GAS}=\left\langle U, \mathfrak{B}, \mathfrak{D}_{\mathfrak{B}}, \mathrm{l}, \mathrm{u}\right\rangle$ be a general approximation space and $S \subseteq U$.

1. If $\mathrm{I}(S)=\mathrm{u}(S)$, then $S$ is a crisp set in GAS.

2. If $S$ and $U \backslash S$ are crisp sets, then $S$ and $U \backslash S$ are totally crisp sets in GAS.

Remark 3 In general case, the members of $\mathfrak{D}_{\mathfrak{B}}$ are not crisp or totally crisp.

Informally, the set $U$ is the universe of approximation; $\mathfrak{B}$ is a nonempty set of base sets, it represents our knowledge used in the whole approximation process; $\mathfrak{D}_{\mathfrak{B}}$ (i.e. the set of definable sets) contains not only the base sets, but those which we want to use to approximate any subset of $U$; the functions I, $\mathrm{u}$ (and b) determine the lower and upper approximation (and the border) of any set with the help of representations of our primitive or available concepts/properties. The nature of an approximation pair ${ }^{3}$ depends on how to relate the lower and upper approximations of a set to the set itself. A general partial approximation space can be specified by giving some requirements for the base set.

\section{Optimistic, average and pessimistic partial membership functions}

Relying on a given general approximation space with Pawlakian approximation pair GAS = $\left\langle U, \mathfrak{B}, \mathfrak{D}_{\mathfrak{B}}, \mathbf{l}, \mathbf{u}\right\rangle$ three different partial membership functions ( $\mu_{s}^{o}$ for optimistic, $\mu_{s}^{a}$ for average and $\mu_{s}^{p}$ for pessimistic) can be introduced. In the definition $^{4}$ we need the neighborhood of $u$ (with respect to $\mathfrak{B}): \mathcal{N}_{\mathfrak{B}}(u)=\{B \mid B \in \mathfrak{B}, u \in B\}$.

Definition 5 Let $S \subseteq U, u \in U$, and $\mathcal{V}_{S}(u)=\left\{\frac{|B \cap S|}{|B|} \mid B \in \mathcal{N}_{\mathfrak{B}}(u)\right\}$.

1. $\mu_{S}^{o}(u)= \begin{cases}\max \left(\mathcal{V}_{S}(u)\right) & \text { if } u \in \cup \mathfrak{B} \\ \text { undefined } & \text { otherwise }\end{cases}$

2. $\mu_{S}^{a}(u)= \begin{cases}\operatorname{avg}\left(\mathcal{V}_{S}(u)\right) & \text { if } u \in \cup \mathfrak{B} \\ \text { undefined } & \text { otherwise }\end{cases}$

3. $\mu_{S}^{p}(u)= \begin{cases}\min \left(\mathcal{V}_{S}(u)\right) & \text { if } u \in \cup \mathfrak{B} \\ \text { undefined } & \text { otherwise }\end{cases}$

For the sake of simplicity it is useful to introduce the crisp membership function $\left(\mu_{S}^{c}\right)$ for any set and any object. It is the partial characteristic function of any set $S$.

Definition 6 If $S(S \subseteq U)$ and $u \in U$, then

\footnotetext{
${ }^{3}$ One of the most general notion of weak and strong approximation pairs can be found in Düntsch and Gediga [12]

${ }^{4}$ Three different functions are used in the definition:

- the function min gives the minimum value of a finite set of numbers;

- the function avr gives the average value of a finite set of numbers, i.e. $\operatorname{avr}\left(\left\{n_{1}, \ldots, n_{k}\right\}\right)=\frac{\Sigma_{i=1}^{k} n_{i}}{k} ;$

- the function max gives the maximum value of a finite set of numbers.
} 


$$
\mu_{S}^{c}(u)= \begin{cases}1 & \text { if } u \in S \\ 0 & \text { if } u \in \cup \mathfrak{B} \backslash S \\ \text { undefined } & \text { otherwise }\end{cases}
$$

Remark 4 If the general approximation space $\mathrm{GAS}=\left\langle U, \mathfrak{B}, \mathfrak{D}_{\mathfrak{B}}, \mathrm{I}, \mathrm{u}\right\rangle$ is total (i.e. $\left.U=\cup \mathfrak{B}\right)$, then all defined membership functions are total.

- $\mu_{S}^{o}(u)=1$ if and only if there is a $B \in \mathcal{N}_{\mathfrak{B}}(u)$ such that $B \subseteq S$, i.e. there is at least one base set which contains $u$, and subset of $S$.

- $\mu_{S}^{o}(u)=0$ if and only if $B \cap S=\emptyset$ for all $B \in \mathcal{N}_{\mathfrak{B}}(u)$.

- $\mu_{S}^{a}(u)=1$ if and only if $B \subseteq S$ for all $B \in$ $\mathcal{N}_{\mathfrak{B}}(u)$.

- $\mu_{S}^{a}(u)=0$ if and only if $B \cap S=\emptyset$ for all $B \in \mathcal{N}_{\mathfrak{B}}(u)$.

- $\mu_{S}^{p}(u)=1$ if and only if $B \subseteq S$ for all $B \in$ $\mathcal{N}_{\mathfrak{B}}(u)$.

- $\mu_{S}^{p}(u)=0$ if and only if there is a $B \in \mathcal{N}_{\mathfrak{B}}(u)$ such that $B \cap S=\emptyset$.

\section{Tool-based partial first-order logic (TbPFoL) relying on different membership functions}

\subsection{Language of TbPFoL}

At first we need a given language of first-order logic, and a finite nonempty set $\mathcal{T}$ of predicate parameters. Its members are called tools.

Definition $7 L$ is a language of TbPFoL with the set $\mathcal{T}$ of tools, if

1. $L=\langle L C$, Var, Con, Term, $\mathcal{T}$, Form $\rangle$

2. $L^{(1)}=\langle L C$, Var, Con, Term, Form $\rangle$ is a language of classical first-order logic;

3. $\mathcal{T} \subseteq \mathcal{P}(1)$, where $\mathcal{P}(1)$ is the set of oneargument predicate parameters;

4. $\mathcal{T}$ is finite and $\mathcal{T} \neq \emptyset$.

The members of set $\mathcal{T}$ are called tools, and their semantic values play a crucial role in giving different types of rough membership functions because they serve as the base of generated approximation space. The semantic values of tools represent available knowledge in a given interpretation.

\subsection{Semantics of TbPFol}

Definition 8 Let $L$ be a language of TbPFoL with the set $\mathcal{T}$ of tools. The ordered pair $\langle U, \varrho\rangle$ is a toolbased interpretation of $L$, if

1. $U$ is a finite nonempty set;

2. $\varrho$ is a function such that $\operatorname{Dom}(\varrho)=$ Con and

(a) if $a \in \mathcal{N}(\mathcal{N}$ is the set of name parameters $)$, then $\varrho(a) \in U$;

(b) if $p \in \mathcal{P}(0)(\mathcal{P}(0)$ is the set of proposition parameters), then $\varrho(p) \in\{0,1\}$; (c) if $P \in \mathcal{P}(n)(n=1,2, \ldots)(\mathcal{P}(n)$ is the set of $n$-argument predicate parameters), then $\varrho(P) \subseteq U^{(n)}$

(d) if $T \in \mathcal{T}$, then $\varrho(T) \neq \emptyset$.

In order to give semantic rules we only need the notions of assignment and modified assignment:

Definition 9 Function $v$ is an assignment relying on the interpretation $\langle U, \varrho\rangle$ if $v: \operatorname{Var} \rightarrow U$.

Definition 10 Let $v$ be an assignment relying on the interpretation $\langle U, \varrho\rangle, x \in V$ ar and $u \in U$. $v[x: u]$ is a modified assignment of $v$, if

1. $v[x: u]:$ Var $\rightarrow U$,

2. $v[x: u](y)=v(y)$ if $x \neq y$, and $v[x: u](x)=u$.

\subsection{Generated tool-based general approximation spaces}

If we have a tool-based interpretation of a language of TbPFoL, then the semantic values of tools (the members of set $\mathcal{T}$ ) determine a general (maybe partial) approximation space with respect to the given interpretation. The generated approximation space is logically relevant in the sense, that it gives the lower and upper approximations (what is more, the different partial membership functions) of any predicate $P$ to be taken into consideration in the definition of semantic rules.

Definition 11 Let $L$ be a language of TbPFoL with the set $\mathcal{T}$ of tools and $\langle U, \varrho\rangle$ be a tool-based interpretation of $L$.

The ordered 5-tuple

$$
\operatorname{GAS}(\mathcal{T})=\left\langle\mathcal{P} \mathcal{R}(U), \mathfrak{B}(\mathcal{T}), \mathfrak{D}_{\mathfrak{B}(\mathcal{T})}, \mathrm{l}, \mathrm{u}\right\rangle
$$

is a logically relevant general partial approximation space generated by set $\mathcal{T}$ of tools with respect to the interpretation $\langle U, \varrho\rangle$ if

1. $\mathcal{P} \mathcal{R}(U)=\bigcup_{n=1}^{\infty} U^{(n)}$, where $U^{(1)}=U, U^{(n)}=U \times U \times \cdots \times U$;

2. $\mathfrak{B}(\mathcal{T})=\bigcup_{n=1}^{\infty} \mathfrak{B}_{n}(\mathcal{T})$ where $\mathfrak{B}_{n}(\mathcal{T})=\left\{\varrho\left(T_{1}\right) \times \cdots \times \varrho\left(T_{n}\right) \mid T_{i} \in \mathcal{T}\right\} ;$

The semantic values of tools (given by the interpretation) generate the set $\mathfrak{B}(\mathcal{T})$. It contains those sets by which the semantic value of any predicate parameter is approximated.

\subsection{Semantic rules of TbPFoL relying on optimistic, average and pessimistic membership function}

In the semantics of TbPFol the semantic value of an expression depends on a given interpretation $I p=\langle U, \varrho\rangle$, a given logically relevant general partial approximation space $\operatorname{GAS}(\mathcal{T})=$ $\left\langle\mathcal{P} \mathcal{R}(U), \mathfrak{B}(\mathcal{T}), \mathfrak{D}_{\mathfrak{B}(\mathcal{T})}, \mathrm{l}, \mathrm{u}\right\rangle$ generated by set $\mathcal{T}$ of 
tools with respect to the interpretation $\langle U, \varrho\rangle$. For the sake of simplicity we use a null entity to represent partiality of semantic rules. We use number 0 for falsity, number 1 for truth, numbers greater than 0 and less than 1 for true degree and number 2 for null entity. In many cases, four possibly different semantic values can be given: optimistic, average, pessimistic and crisp ones. The forms of semantic rules are similar in different cases and so the superscript * can be used to denote one of them $\left.{ }^{\star} \in\left\{{ }^{o},{ }^{a}, p,{ }^{c}\right\}\right)$. The semantic value of an expression $A$ with respect to $I p=\langle U, \varrho\rangle, \operatorname{GAS}(\mathcal{T})$ and the assignment $v$ is denoted by $\llbracket A \rrbracket_{v}^{\star}$.

The most important semantic rules are the following:

1. If $T \in \mathcal{T}$, i.e. $T$ is a tool and $t \in T e r m$, then

$$
\llbracket T(t) \rrbracket_{v}^{\star}=\left\{\begin{array}{ll}
2 & \text { if } \llbracket t \rrbracket_{v}^{\star} \notin \cup \mathfrak{B} ; \\
1 & \text { if } \llbracket t \rrbracket_{v}^{\star} \in \varrho(T) ; . \\
0 & \text { otherwise. }
\end{array} .\right.
$$

2. If $P \in P(n)(n \neq 0), P \notin \mathcal{T}$, i.e. $P$ is an $\mathrm{n}-$ argument predicate parameter which is not a tool and $t_{1}, t_{2}, \ldots, t_{n} \in$ Term, then

$$
\llbracket P\left(t_{1}, \ldots, t_{n}\right) \rrbracket_{v}^{\star}=\mu_{\varrho(P)}^{\star}\left(\left\langle\llbracket t_{1} \rrbracket_{v}^{\star}, \ldots, \llbracket t_{n} \rrbracket_{v}^{\star}\right\rangle\right) .
$$

3. If $A \in$ Form, then

$$
\llbracket \neg A \rrbracket_{v}^{\star}= \begin{cases}2 & \text { if } \llbracket A \rrbracket_{v}^{\star}=2 \\ 1-\llbracket A \rrbracket_{v}^{\star} & \text { otherwise }\end{cases}
$$

4. If $A, B \in$ Form, then

$$
\begin{aligned}
& \llbracket(A \wedge B) \rrbracket_{v}^{\star}= \\
& = \begin{cases}2 & \text { if } \llbracket A \rrbracket_{v}^{\star}=2 ; \\
2 & \text { if } \llbracket B \rrbracket_{v}^{\star}=2 ; \\
\min \left(\left\{\llbracket A \rrbracket_{v}^{\star}, \llbracket B \rrbracket_{v}^{\star}\right\}\right), & \text { otherwise }\end{cases} \\
& \llbracket(A \vee B) \rrbracket_{v}^{\star}=\max \left(\left\{\llbracket A \rrbracket_{v}^{\star}, \llbracket B \rrbracket_{v}^{\star}\right\}\right) \\
& \llbracket(A \supset B) \rrbracket_{v}^{\star}= \\
& = \begin{cases}2 & \text { if } \llbracket A \rrbracket_{v}^{\star}=2 ; \\
2 & \text { if } \llbracket B \rrbracket_{v}^{\star}=2 ; \\
\min \left(\left\{1,1-\llbracket A \rrbracket_{v}^{\star}+\llbracket B \rrbracket_{v}^{\star}\right\}\right), & \text { otherwise }\end{cases}
\end{aligned}
$$

5. If $A \in$ Form, $x \in \operatorname{Var}$ and

$$
\begin{aligned}
& \mathcal{V}^{\star}(A)=\left\{\llbracket A \rrbracket_{v[x: u]}^{\star} \mid u \in U\right\}, \text { then } \\
& \llbracket \forall x A \rrbracket_{v}^{\star}=\min \left(\mathcal{V}^{\star}(A)\right) \\
& \llbracket \exists x A \rrbracket_{v}^{\star}= \begin{cases}2 & \text { if for all } u \in U: \\
\max \left(\mathcal{V}^{\star}(A)\right), & \text { otherwise }\end{cases}
\end{aligned}
$$

\section{Different notions of logical consequences}

From the logical point of view, flexibility is the main advantage of our logical framework. It can be recognized on different levels:

1. The generated partial interpretations rely on two theoretical points:

(a) the set of semantic values of tools given by the total interpretation $U$. These semantic values represent available knowledge, i.e. the total interpretation gives us the representations of available concepts/properties and relations by which we approximate any concept/property or relation (with respect to the given interpretation);

(b) the general partial approximation space generated by tools with respect to the given interpretation.

2. In a consequence relation the diffeent membership functions can be used for different formulae. So the consequence relation can rely on possibly different semantic values determined by total, optimistic, average and pessimistic membership functions and so the investigations of different decisions (based on different membership functions) are possible.

The notion of models plays a fundamental role in the semantic definition of consequence relation:

Definition 12 Let $L$ be a language of TbPFoL with the set $\mathcal{T}$ of tools, $\Gamma=\left\langle A_{1}, A_{2}, \ldots, A_{n}\right\rangle$ be an ordered $n$-tuple of closed formulae $\left(A_{1}, A_{2}, \ldots, A_{n} \in\right.$ Form) and $\mathcal{I}$ is a nonempty set of interpretation of $L$.

1. The ordered $n$-tuple $\Delta=\left\langle\delta_{1}, \ldots, \delta_{n}\right\rangle$ is a decision type of $\Gamma$ if $\delta_{1}, \ldots, \delta_{n} \in\{o, a, p, c\}$.

2. Let $\Delta=\left\langle\delta_{1}, \ldots, \delta_{n}\right\rangle$ be a decision type $\Gamma$. Then

(a) $\langle U, \varrho, v\rangle$ is a $\Delta$-type model of $\Gamma$ with respect to $\mathcal{I}$ if

i. $\langle U, \varrho\rangle \in \mathcal{I}$;

ii. $v$ is an assignment relying on $\langle U, \varrho\rangle$; iii. $\llbracket A_{i} \rrbracket_{v}^{\delta_{i}}=1$ for all $i(i=1,2, \ldots, n)$.

(b) $\langle U, \varrho, v\rangle$ is a $\Delta$-type model of $\Gamma$ with parameter $\alpha(0<\alpha \leq 1)$ with respect to $\mathcal{I}$, if

i. $\langle U, \varrho\rangle \in \mathcal{I}$

ii. $v$ is an assignment relying on $\langle U, \varrho\rangle$; iii. $\llbracket A_{i} \rrbracket_{v}^{\delta_{i}} \neq 2$ for all $i(i=1,2, \ldots, n)$ iv. $\llbracket A_{i} \rrbracket_{v}^{\delta_{i}} \geq \alpha$ for all $i(i=1,2, \ldots, n)$.

(c) $\langle U, \varrho, v\rangle$ is a $\Delta$-type partial model of $\Gamma$ with parameter $\alpha(0<\alpha \leq 1)$ with respect to $\mathcal{I}$ if

i. $\langle U, \varrho\rangle \in \mathcal{I}$;

ii. $v$ is an assignment relying on $\langle U, \varrho\rangle$; iii. $\llbracket A_{i} \rrbracket_{v}^{\delta_{i}} \geq \alpha$ for all $i(i=1,2, \ldots, n)$.

Definition 13 Let $L$ be a language of TbPFoL with the set $\mathcal{T}$ of tools, $\Gamma=\left\langle A_{1}, A_{2}, \ldots, A_{n}\right\rangle$ be an ordered $n$-tuple of closed formulae $\left(A_{1}, A_{2}, \ldots, A_{n} \in\right.$ Form), $B \in$ Form be a closed formula and $\mathcal{I}$ is a nonempty set of interpretation of $L$.

1. $\Delta \rightarrow \delta$ is a decision driven consequence type from $\Gamma$ to $B$ if

(a) $\Delta$ is a decision type of $\Gamma$;

(b) $\delta$ is a decision type of $B$.

2. Let $\Delta \rightarrow \delta$ is a decision driven consequence type from $\Gamma$ to $B$. 
(a) $B$ is a strong consequence of $\Gamma$ driven by $\Delta \rightarrow \delta$ with respect to $\mathcal{I}$ if all $\Delta-$ type models of $\Gamma$ are $\delta$-type models of $B$ $\left(\Gamma \models_{\Delta \rightarrow \delta}^{s} B\right)$.

(b) $B$ is a parametrized consequence of $\Gamma$ driven by $\Delta \rightarrow \delta$ with the parameter pair $\langle\alpha, \beta\rangle$ with respect to $\mathcal{I}$ if all $\Delta$-type models of $\Gamma$ with the parameter $\alpha$ are $\delta$ type models of $B$ with the parameter $\beta$ $\left(\Gamma \vDash_{\Delta \rightarrow \delta}^{\langle\alpha, \beta\rangle} B\right)$.

(c) $B$ is a partial parametrized consequence of $\Gamma$ driven by $\Delta \rightarrow \delta$ with the parameter pair $\langle\alpha, \beta\rangle$ with respect to $\mathcal{I}$ if all $\Delta$-type partial models of $\Gamma$ with the parameter $\alpha$ are $\delta$-type partial models of $B$ with the parameter $\beta\left(\Gamma \models_{\Delta \rightarrow \delta}^{p,\langle\alpha, \beta\rangle} B\right)$.

Next theorem shows some important connections between different notions of consequence relations.

- $\mathcal{I}_{t}$ is the set of interpretation such that the generated general approximation space is a total (or covering) one:

$\mathcal{I}_{t}=\left\{\langle U, \varrho\rangle \mid U=\cup_{T \in \mathcal{T}} \varrho(T)\right\}$

- $\mathcal{I}_{d}$ is the set of interpretation such that the generated approximation space is one-layered:

$\mathcal{I}_{d}=\left\{\langle U, \varrho\rangle \mid \varrho(T) \cap \varrho\left(T^{\prime}\right)=\emptyset, T \neq T^{\prime}\right\}$

Theorem 14 Let $L$ be a language of TbPFoL with the set $\mathcal{T}$ of tools, $A_{1}, A_{2}, \ldots, A_{n}, B$ be closed formulae containing only tools as predicate parameters and $\Gamma=\left\langle A_{1}, A_{2}, \ldots, A_{n}\right\rangle$.

1. If $\Gamma \vDash_{\langle c, \ldots, c\rangle \rightarrow c}^{s} B$ with respect to $\mathcal{I}_{t}$, then

(a) $\Gamma \vDash_{\langle p, \ldots, p\rangle \rightarrow p}^{s} B$ with respect to $\mathcal{I}_{t}$;

(b) $\Gamma \vDash_{\langle o, \ldots, o\rangle \rightarrow o}^{s} B$ with respect to $\mathcal{I}_{t}$.

2. The following four consequence relations are equivalent with respect to $\mathcal{I}_{t} \cap \mathcal{I}_{d}$ :
(a) $\Gamma \vDash{ }_{\langle c, \ldots, c\rangle \rightarrow c}^{s} B$
(b) $\Gamma \vDash{ }_{\langle o, \ldots, o\rangle \rightarrow o}^{s} B$
(c) $\Gamma \models_{\langle a, \ldots, a\rangle \rightarrow a}^{s} B$
(d) $\Gamma \vDash_{\langle p, \ldots, p\rangle \rightarrow p}^{s} B$

Theorem 15 Let $\delta \in\{o, a, p, c\}$.

1. If $0<\beta \leq \alpha \leq 1$ then $\{A \supset B, A\} \vDash_{\langle\delta, \delta\rangle \rightarrow \delta}^{\langle\alpha, \beta\rangle} B$.

2. If $\alpha=1,0<\beta \leq 1,\{A \supset B, A\} \vDash_{\langle\delta, \delta\rangle \rightarrow \delta}^{\langle 1, \beta\rangle} B$.

3. $\{A \supset B, A\} \vDash_{\langle\delta, \delta\rangle \rightarrow \delta}^{s} B$.

\section{Conclusion}

If we want to investigate decision theoretic rough set models from the logical point of view or we want to apply its results in inferences, we have to face a certain problem. What happens if in the semantics of first-order logic we use optimistic, average and pessimistic partial membership functions as the semantic values of predicate parameters instead of total characteristic functions given by their total interpretation? In this paper the semantic system of a partial first-order logic with three different types of partial membership functions is presented. The semantics relies on a very general notion of approximation spaces generated by available knowledge (appearing in properties), and so it gives a very flexible common framework of different systems of set approximation. By using two notions of parametrized consequence relations the logical consequences of decisions based on decision theoretic rough set models can be investigated. The next step has to be made in future is to give the most important logical laws of the presented logical system.

Open questions:

- Can Pawlak's original decision logic be interpreted in the given logical system?

- How can some important concepts known in the theory of rough sets (e.g. reduct and superreduct) be expressed in the introduced logical framework?

\section{Acknowledgements}

The publication was supported by the TÁMOP4.2.2.C-11/1/KONV-2012-0001 project. The project has been supported by the European Union, co-financed by the European Social Fund.

\section{References}

[1] Pawlak, Z.: Rough sets. International Journal of Information and Computer Science 11(5) (1982) 341-356

[2] Pawlak, Z.: Rough Sets: Theoretical Aspects of Reasoning about Data. Kluwer Academic Publishers, Dordrecht (1991)

[3] Yao, Y.Y.: On generalizing rough set theory. In: Proceedings of the 9th International Conference Rough Sets, Fuzzy Sets, Data Mining, and Granular Computing (RSFDGrC 2003). LNAI 2639, Springer-Verlag (2003) 44-51

[4] Yao, Y.Y.: Information granulation and rough set approximation. International Journal of Intelligent Systems 16(1) (2001) 87-104

[5] Yao, Y.Y.: Decision-theoretic rough set models, In: Rough Sets and Knowledge Technology, Second International Conference, RSKT 2007, Proceedings, LNAI 4481, Springer-Verlag (2007) $1-12$

[6] Skowron, A., Stepaniuk, J.: Information granules: Towards foundations of granular computing. International Journal of Intelligent Systems 16(1) (2001) 57-85

[7] Skowron, A., Świniarski, R., Synak, P.: Approximation spaces and information granulation. LNCS Transactions on Rough Sets $\mathbf{3 4 0 0 ( 3 )}$ (2005) 175-189

[8] Zadeh, L.A.: Granular computing and rough set theory. In Kryszkiewicz, M., Peters, J.F., Rybin- 
ski, H., Skowron, A., eds.: Rough Sets and Intelligent Systems Paradigms, International Conference, RSEISP 2007, Warsaw, Poland, June 2830, 2007, Proceedings. Volume 4585 of Lecture Notes in Computer Science., Springer (2007) 14

[9] Lin, T.Y.: Approximation theories: Granular computing vs rough sets. In Chan, C.C., Grzymala-Busse, J.W., Ziarko, W., eds.: Rough Sets and Current Trends in Computing, 6th International Conference, RSCTC 2008, Akron, OH, USA, October 23-25, 2008, Proceedings. Volume 5306 of Lecture Notes in Computer Science., Springer (2008) 520-529

[10] Pawlak, Z., Polkowski, L., Skowron, A.: Rough sets: An approach to vagueness. In Rivero, L.C., Doorn, J., Ferraggine, V., eds.: Encyclopedia of Database Technologies and Applications. Idea Group Inc., Hershey, PA (2005) 575-580

[11] Zhu, P.: Covering rough sets based on neighborhoods: An approach without using neighborhoods. International Journal of Approximate Reasoning 52(3) (March 2011) 461-472

[12] Düntsch, I., Gediga, G.: Approximation operators in qualitative data analysis. In de Swart, H.C.M., Orlowska, E., Schmidt, G., Roubens, M., eds.: Theory and Applications of Relational Structures as Knowledge Instruments. Volume 2929 of Lecture Notes in Computer Science., Springer (2003) 214-230

[13] Pawlak, Z., Skowron, A.: Rudiments of rough sets. Information Sciences 177(1) (2007) 3-27

[14] Polkowski, L.: Rough Sets: Mathematical Foundations. Advances in Soft Computing, Physica-Verlag, Heidelberg, 2002.

[15] Csajbók, Z., Mihálydeák, T.: Partial approximative set theory: A generalization of the rough set theory. International Journal of Computer Information System and Industrial Management Applications 4 (2012) 437-444

[16] Csajbók, Z., Mihálydeák, T.: A General Set Theoretic Approximation Framework. In: Greco, S., Bouchon-Meunier, B., Coletti, G., Fedrizzi, M., Matarazzo, B., Yager R. R. (eds): Proceedings of IPMU 2012, Catania, Italy, July 9-13, 2012, Part I, CCIS, Volume 297, Springer (2012) 604-612

[17] Mihálydeák, T.: Partial first-order logical semantics based on approximations of sets. In: Cintula, P., Ju, S., Vita, M. (eds.): Non-classical Modal and Perdicate Logics 2011, Guangzhou (Canton), China, F solutions, Prague (2011), 85-90.

[18] Mihálydeák, T.: Partial first-order logic with approximative functors based on properties. In: Li, T., Nguyen, H.S., Wang, G., GrzymalaBusse, J., Janicki, R., Hassanien, A.E., Yu, H. (eds.): Rough Sets and Knowledge Technology. 7th International Conference, RSKT 2012, Chengdu, China, August 17-20, 2012, Proceed- ings. LNAI, vol. 7414, Springer (2012) 514-523 